\title{
Approach For Cardiac Action Potential Detection From Noised Recordings
}

\author{
N. Ivanushkina \\ Department of Electronic Engineering \\ Igor Sikorsky Kyiv Polytechnic Institute \\ Kyiv, Ukraine \\ n.ivanushkina@gmail.com \\ A. Redaelli \\ Politecnico di Milano \\ Milan, Italy \\ alberto.redaelli@polimi.it
}

\author{
K. Ivanko \\ Department of Electronic Engineering \\ Igor Sikorsky Kyiv Polytechnic Institute \\ Kyiv, Ukraine \\ koondoo@gmail.com \\ V.Timofeyev \\ Department of Electronic Engineering \\ Igor Sikorsky Kyiv Polytechnic Institute \\ Kyiv, Ukraine \\ timofkpi@gmail.com
}

\author{
Y. Prokopenko \\ Department of Electronic Engineering \\ Igor Sikorsky Kyiv Polytechnic Institute \\ Kyiv, Ukraine \\ prok@ee.kpi.ua \\ M. Ivanushkina \\ Bogomolets National Medical \\ University \\ Kyiv, Ukraine \\ ivanushkina.mmasha@gmail.com
}

\begin{abstract}
The morphology of action potential (AP) is a key element in the determination of normal or pathological processes in the cardiac cells and the diagnosis of heart diseases. Therefore obtaining useful information about the shape of AP pulses minimizing the noise contribution is an important task of signal processing to improve the diagnosis process. In this paper a method to detect the APs of cardiomyocytes is proposed, based on wavelet transform, decomposition and reconstruction in eigenvectors basis of cardiac cycles. The parallel conductance model is used to simulate the cardiac AP and ion currents taking into account white or pink noise. Numerical experiments demonstrate the performance of the AP detection for various signal-to-noise ratio (SNR). The proposed method can be used to obtain undistorted AP morphologies in order to interpret experimental studying of APs functional properties in cardiomyocytes by means of lab-on-chip technologies.
\end{abstract}

Keywords - action potential; cardiomyocyte; parallel conductance model; wavelet analysis; decomposition in eigenvectors basis, lab-on-chip technology, human-induced pluripotent stem cells.

\section{INTRODUCTION}

Heart diseases continue to remain the most widespread human diseases. Many scientists focus their efforts to study the heart activity by using experimental and mathematical models $[1,2]$. One of the directions in cardiac cellular-level experimental studies is the use of lab-on-chip platforms, which is rapidly growing [3,4]. Heart-on-chip technology is advanced in vitro tool to study the human-induced pluripotent stem cells (hiPSCs) differentiated into cardiomyocytes (hiPSC-CMs). Through heart-on-chip technology researchers successfully modeled a human heart tissue on an engineered chip for study of heart diseases, drug screening and cell-replacement therapies, contemporarily reducing the need for human and animal testing.

Different electrophysiological technologies are used to investigate the functional properties of hiPSC-CMs. Works $[5,6]$ are devoted to a classical approach to record intracellular electrical activity, based on patch-clamp technique, and to multielectrode potential recordings for the study of ion channels functions. Contactless imaging methods are performed by using voltage- and calciumsensitive fluorescent dyes [7]. Multicellular recordings, obtained by these techniques, have high spatial resolution of transmembrane potential and intracellular calcium transients for studying cardiomyocytes differentiation and drug discovery.
Numerous investigators have analyzed the shape of APs to estimate the state and functionality of the cardiac cells. Different morphologies of cardiac AP allow for evaluating the degree of maturation of hiPSC-CMs and their transformation to the main types: nodal-like, atrial-like, and ventricular-like cells. It is shown in [5], that cardiomyocyte development and functional maturity are characterized by the change of AP properties. In agreement with [8] studies on cardiac beating rhythm are typically based on the detection and assessment of AP maximal rate of depolarization and repolarization. In [9] authors have demonstrated the influence of different factors on hiPSC-CMs and their effectiveness on the evaluation of cardiac cells functionality based on the change of AP morphologies. In addition, methods of drug screening are performed using AP changes in cardiac cells in the study [3].

To create a native myocardial tissue, a technology based on heart-on-chip platform has been recently developed, which recapitulate the $3 \mathrm{D}$ architecture of complex cell-cell interactions [10]. The proposed device (Fig.1) allows for performing electrical and mechanical stimulation to study the cardiac excitation-contraction coupling by evaluating the excitation threshold, maximum capture rate and amplitude of contractions in cardiomyocytes.

However, experimental studies of hiPSC-CMs are complex and along with advantages have limitations, related to the maintenance of the environmental conditions. In addition, signals of functional activity, recorded from cardiac cells, are characterized by varying degrees of noise.

Thus, for the investigation of cardiac electrical activity, as well as the assessment of normal or pathological processes in cardiac cells aimed at revealing of heart diseases, it is necessary to obtain diagnostically important information about AP morphology minimizing the noise contribution. Despite the theoretical importance of this process, not many works are devoted to AP detection from noised recordings with low SNR.

The present study is devoted to improvement of methods for research of hiPSC-CMs' electrical activity by computational modeling and signal processing for noise elimination and detection of AP patterns.

\section{ACTION Potentials AND NoISE SimUlation}

It is known, that generation and propagation of AP reflect bioelectric processes occurring in the heart cells. The genesis and formation of AP are determined by main ionic currents 
of cardiomyocytes for sodium, potassium and calcium ions, which are characterized by passing through channels with noise.

In this study, the model of electrical activity in cardiomyocytes [11] is used to simulate the dynamics of transmembrane potential and currents taking into account the noise. Due to the fact that independent conductance channels are considered for $\mathrm{K}^{+}, \mathrm{Na}^{+}, \mathrm{Ca}^{2+}$, as well as leakage using the Hodgkin-Huxley approach [1], this model allows for describing various noise types by including additional current source in the parallel branches. There are three main types of noise in excitable membranes: thermal noise, shot noise and flicker noise.

In accordance with the parallel conductance model, the alternative component of the membrane potential $v_{m}(t)$ under the influence of the depolarizing current $I_{d}$ can be determined from the differential equation

$$
\left.\frac{d v_{m}}{d t}=\frac{1}{C_{m}}\left(-I_{K}\left(v_{m}, t\right)-I_{N a}\left(v_{m}, t\right)\right)-I_{C a}\left(v_{m}, t\right)-I_{l}+I_{d}+I_{N}\right)
$$

where $I_{K}, I_{N a}, I_{C a}$ are currents for $\mathrm{K}^{+}, \mathrm{Na}^{+}, \mathrm{Ca}^{2+}$ ions, $I_{l}$ is current of the leakage through the membrane, $I_{d}$ is the depolarization current, and $I_{N}$ is noise component of the current.

To simulate noise appearance, the additional noise component of current is added into the equation. Noise component reflects sources of noised ion currents in the cell membrane. Most often $[12,13]$ this component is described using by white noise, which is produced by Gaussian random numbers with given variance (Fig. 2, a).

According to the theory [1], cardiac AP is formed by currents for $\mathrm{K}^{+}, \mathrm{Na}^{+}, \mathrm{Ca}^{2+}$ ions, which are characterized by the different relaxation degree of activation and inactivation

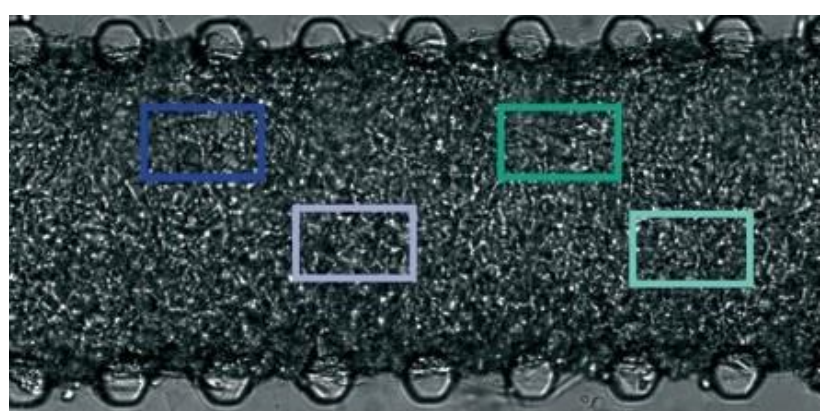

a)

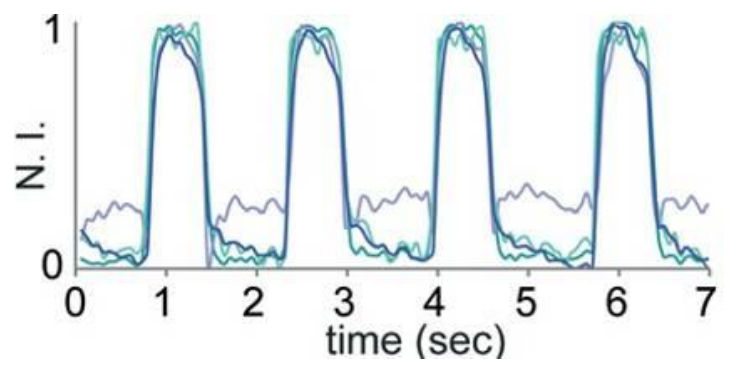

b)

Fig. 1. Research of cardiac cells activity by means of 3D heart-on-chip microdevice: $a$ ) monitoring in three different areas (rectangles) of construction (cardiac cells, suspended in the matrix of fibrin gel) under stimulated conditions; $b$ ) experimental results of functionality of cardiac cells. Modified from [10].

functions, and the slowest processes occur in $\mathrm{Ca}^{2+}$ channels. So it can be assumed, that currents for $\mathrm{Ca}^{2+}$ ions play the most significant role in the noise processes taking place with decreasing frequency of AP generation. In this case, the

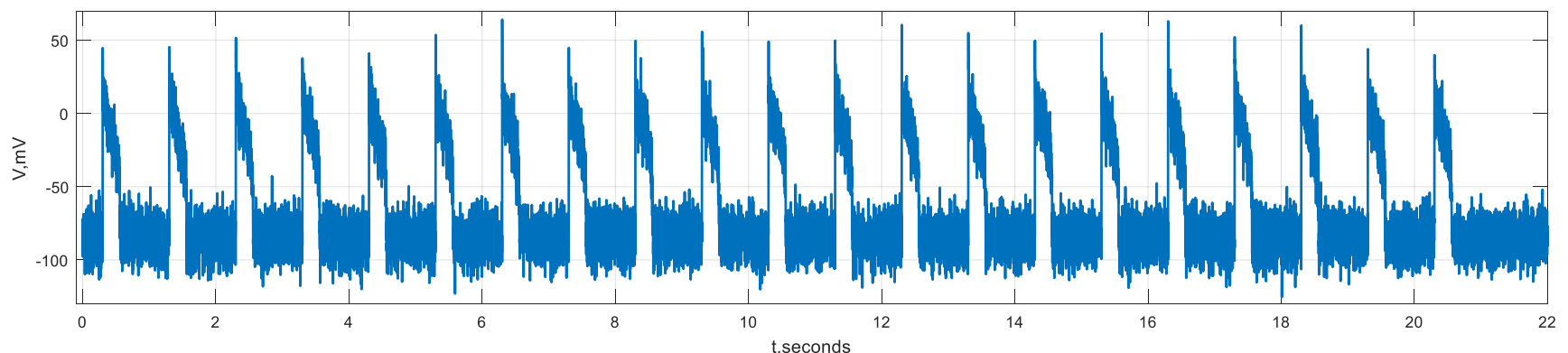

a)

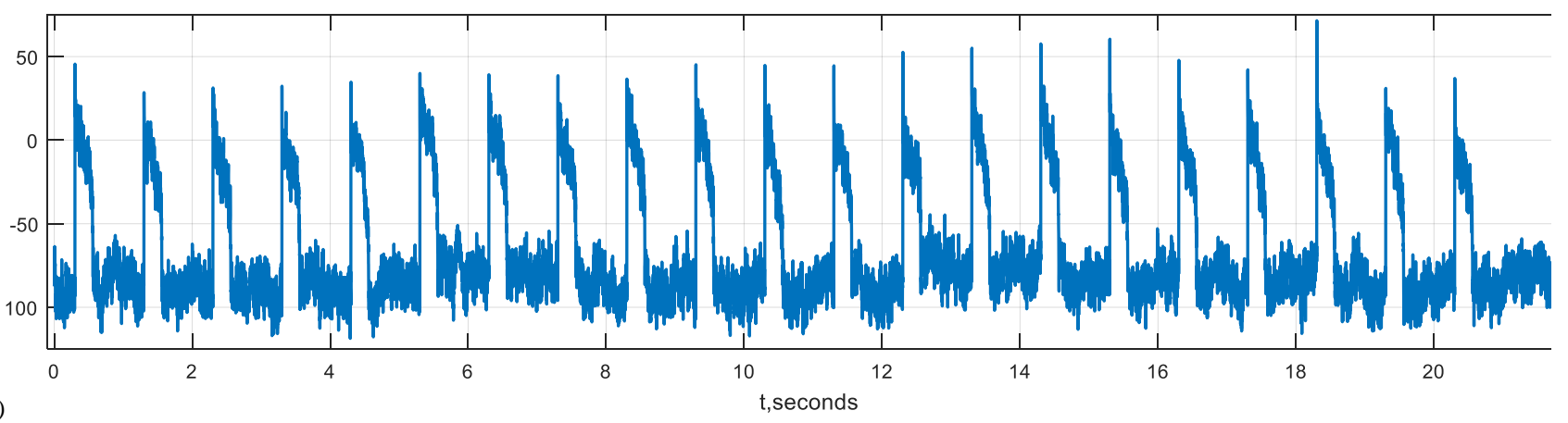

Fig. 2. Simulation of action potentials sequence obtained as replies of cardiomyocytes to 20 stimuli at constant stimulation frequency Fst $=1$ Hz (cycle length $\mathrm{CL}=1000 \mathrm{~ms}$ ). Sample frequency of the simulated signals is $1 \mathrm{kHz} . a$ ) corrupted by white noise; $b$ ) corrupted by pink (flicker) noise 
spectral density for current pink noise (Fig. 2, b), measured under voltage clamp conditions, is proportional to the square of the mean current, and inversely proportional to the frequency [13]:

$$
W_{N}(f)=\frac{A}{N_{t}} \frac{I^{2}}{f}
$$

where $W_{N}(f)$ is the spectral density for current noise, $N_{t}$ is the total number of charge carriers within the membrane, $I$ is the mean current, $f$ is the frequency, and $A$ is a constant equal about 1 for ions in salt solutions.

\section{METHOD FOR APS EXTRACTION IN NOISY RECORDINGS}

Many researchers have collected data set on the processing of weak biosignals for identifying, analyzing and displaying of low-amplitude components [14]. The importance of AP detection and pattern recognition in nerve tissues is reflected in [15-17].

In this paper, a method is proposed to detect APs in noisy recordings in cardiac cells. A similar approach to signal processing of low-amplitude potentials was used by us in high-resolution ECG systems [14]. The complex method combines wavelet transform and procedures of decomposition and reconstruction in eigenvectors basis.

The suggested method includes the following steps:

- accumulation of the AP signals synchronized by the excitation pulses (Fig. 3);

- wavelet analysis of the AP signals, highlighting the detail levels with noise components;

- removing noise by thresholding of detail coefficients of selected levels;

- de-noising of the AP signals by reconstruction in the wavelet basis without noise components;

- decomposition of the array of reconstructed AP in the basis of eigenvectors, the selection of the principal eigenvectors;

- reconstruction of the original AP signals in the basis of principal eigenvectors;

- formation of AP features for pattern recognition.

Wavelet decomposition is performed so that each AP in an ensemble of cycles corresponds to a set of wavelet coefficients of the approximation and detail levels. To remove the noise, selection of the threshold of discarded detail coefficients of each level is made.

For processing in the basis of eigenvectors the whole recording of APs signals can be represented as an ensemble of separately taken realizations of action potentials synchronized by an excitation pulse:

$$
\begin{aligned}
& E_{1}=e_{11}, e_{12}, \ldots, e_{1 n} \\
& E_{2}=e_{21}, e_{22}, \ldots, e_{2 n}, \\
& \vdots \\
& E_{m}=e_{m 1}, e_{m 2}, \ldots, e_{m n}
\end{aligned}
$$

where $E_{1}, E_{2}, \ldots E_{m}$ is the ensemble of $m$ realizations of AP signals; $\quad e_{i j}(i, j=\overline{1, n})$ are discrete values of each realization; $m$ is the number of signal realizations.

The covariance matrix of the ensemble of realizations of APs can be presented as follows:

$$
C=\left[\begin{array}{cccc}
C_{11} & C_{12} & \ldots & C_{1 n} \\
C_{21} & C_{22} & \ldots & C_{2 n} \\
\vdots & & & \\
C_{n 1} & C_{n 2} & \ldots & C_{n n}
\end{array}\right]
$$

where the elements $C_{q r}$ of the matrix $C$ can be represented as:

$$
C_{q r}=\sum_{p=1}^{m}\left(e_{p q}-\bar{e}_{p}\right)\left(e_{p r}-\bar{e}_{p}\right),
$$

where $\bar{e}_{p}$ is the mean value for the realization $p$.

The problem of determining eigenvalues and eigenvectors can be interpreted as an algebraic analogue of finding solutions to a system of ordinary differential equations. In this case, the eigenvalues of the matrix $\lambda_{1} \geq \lambda_{2} \geq \ldots \geq \lambda_{n}$ are found as the roots of the characteristic equation of the covariance matrix $C$, and the eigenvectors $V_{1}, V_{2} \ldots, V_{n}$ representing the orthonormal basis of the

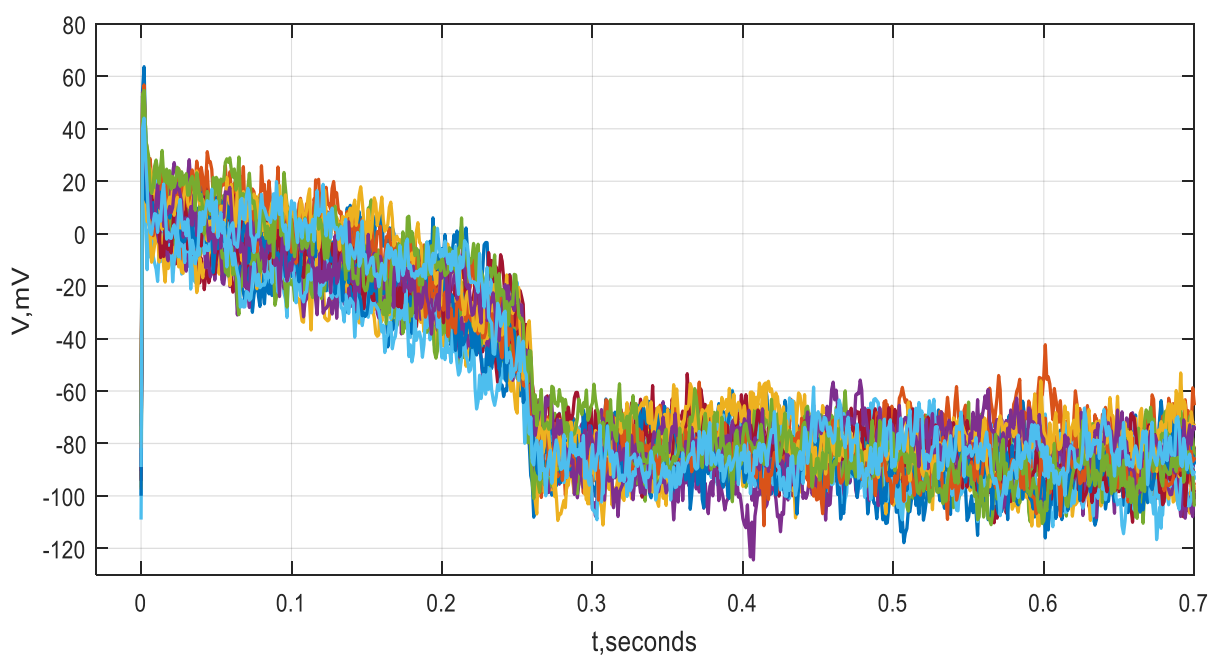

Fig. 3. Accumulation of 20 simulated cardiac action potentials, corrupted by pink (flicker) noise, with synchronization by the excitation pulses $\left(F_{s t}=1 \mathrm{~Hz}\right)$ 
ensemble of AP cycles are determined from the system of linear algebraic equations:

$$
C V=\lambda V
$$

After determining the eigenvectors $V_{1}, V_{2} \ldots, V_{n}$ and the eigenvalues $\lambda_{1} \geq \lambda_{2} \geq \ldots \geq \lambda_{n}$ of the covariance matrix of the restored AP signal, the sorting (in descending order) of the eigenvalues and the choice of several principal eigenvectors corresponding to the most important eigenvalues are made. In the resulting basis, the principal eigenvectors $V_{1}, V_{2} \ldots, V_{\text {s }}$ contain the main information about the original ensemble of AP signals.

The projections $b_{i j}$ in the basis of the principal eigenvectors $V_{1}, V_{2} \ldots, V_{s}$ are determined by the scalar product of the selected AP signal realizations on the coordinate axes:

$$
b_{i j}=\left(E_{i} \cdot V_{j}\right)=\sum_{k=1}^{s} E_{i k} \cdot V_{i k} .
$$

Reconstruction in the coordinate basis of the principal eigenvectors is performed according to the expression:
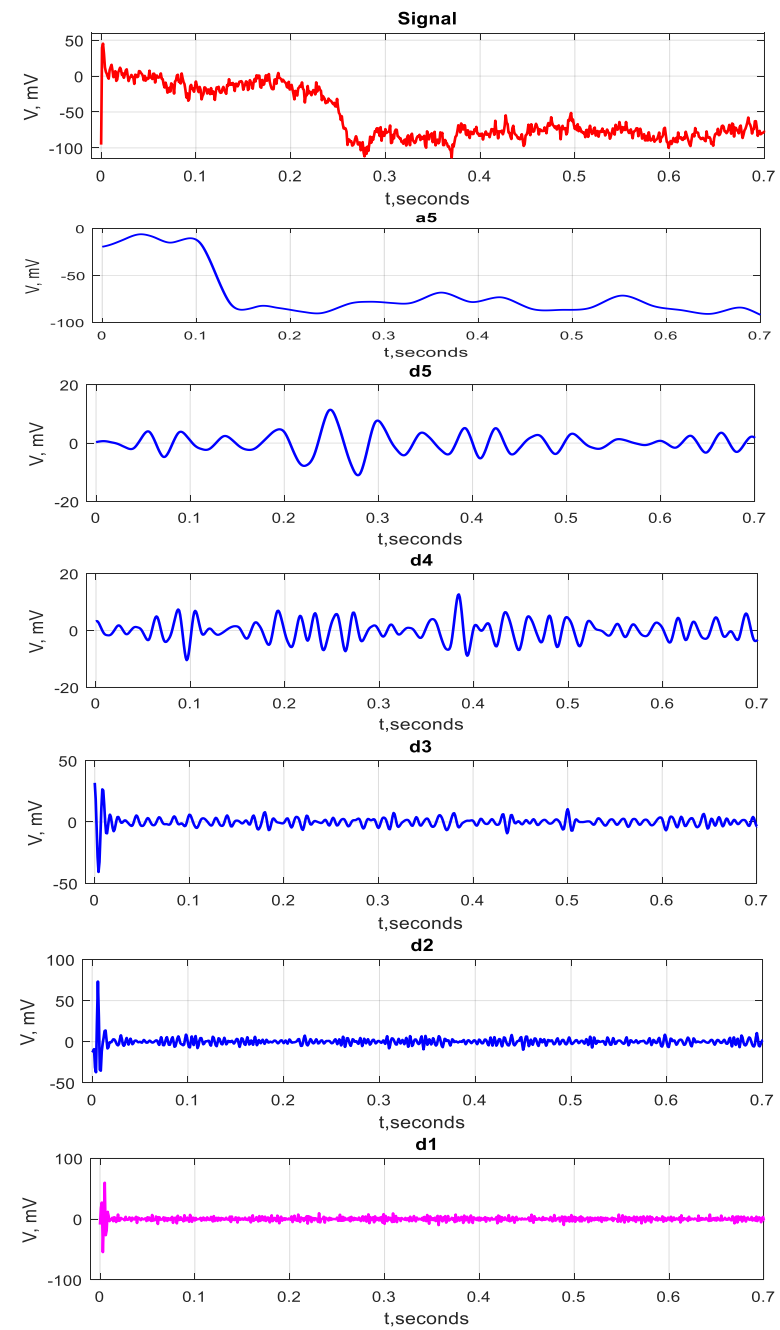

Fig. 4. Wavelet decomposition to the $5^{\text {th }}$ level with using a "symmetric" wavelet of $6^{\text {th }}$ order performed for the simulated AP corrupted by pink noise with $\mathrm{SNR}=10 \mathrm{~dB}$

$$
E_{i}^{*}=\sum_{j=1}^{s} b_{i j} V_{j}
$$

The numerical values of projections $b_{i j}$ reflect the information contained in the AP signal and may be diagnostic parameters in the study of electrical activity of the heart, in particular, the diagnostic features for AP classification and pattern recognition.

\section{RESULTS AND DISCUSSION}

To simulate electrical activity in cardiac cells, numerical experiments were performed in Matlab environment using the parallel conductance model. The processes of AP generation and changes of currents for $\mathrm{K}^{+,} \mathrm{Na}^{+}, \mathrm{Ca}^{2+}$ channels, as well as electrophysiological properties in ventricular cardiomyocytes were investigated taking into account 2 types of noise: white and pink (flicker).

The goal of our study was the simulation of the dynamics of cardiac electrical activity under the influences of white and pink types of noise and creation of methods for detection of AP from noisy recordings.

In this work, the standard protocol of electrical stimulation was used. The set of 20 APs (20 replies of cardiomyocytes from the 20 stimuli) at constant stimulation frequency $F_{s t}=1 \mathrm{~Hz}$ or cycle length $C L=1000 \mathrm{~ms}$ was simulated and corrupted by white noise and pink noise. Sample frequency of the simulated signals is $1 \mathrm{kHz}$.

The approximation coefficients of multilevel discrete wavelet analysis reflect the low-frequency components of APs and the detail coefficients represent high-frequency components (Fig. 4). Based on this, wavelet decomposition can be widely used to eliminate noise, which appears at the detail levels of the wavelet transform. Noise suppression in APs was performed by detail coefficients processing. The APs sequences were decomposed to the 5th level with a "symmetric" wavelet of 6th order. Taking into account the pattern of white and pink noise at these levels, it was reasonable to use the universal thresholds for thresholding implementation and apply the thresholds in a scaledependent manner. Then, using the thresholded coefficients for the inverse wavelet transformation, the APs sequences
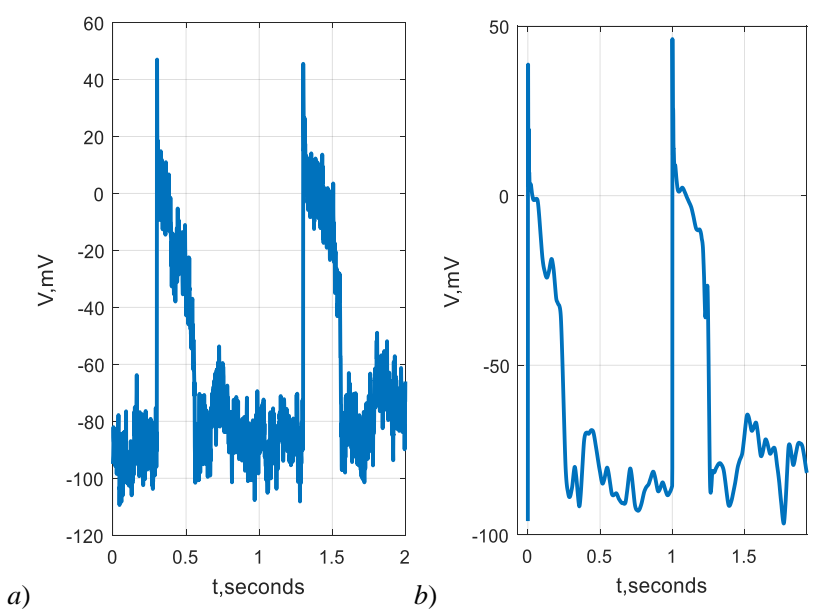

Fig. 5. Results of APs processing using wavelet transform: $a$ ) original APs with background pink noise, $\mathrm{SNR}=10 \mathrm{~dB} ; b)$ denoised APs with distortions and residual noise 

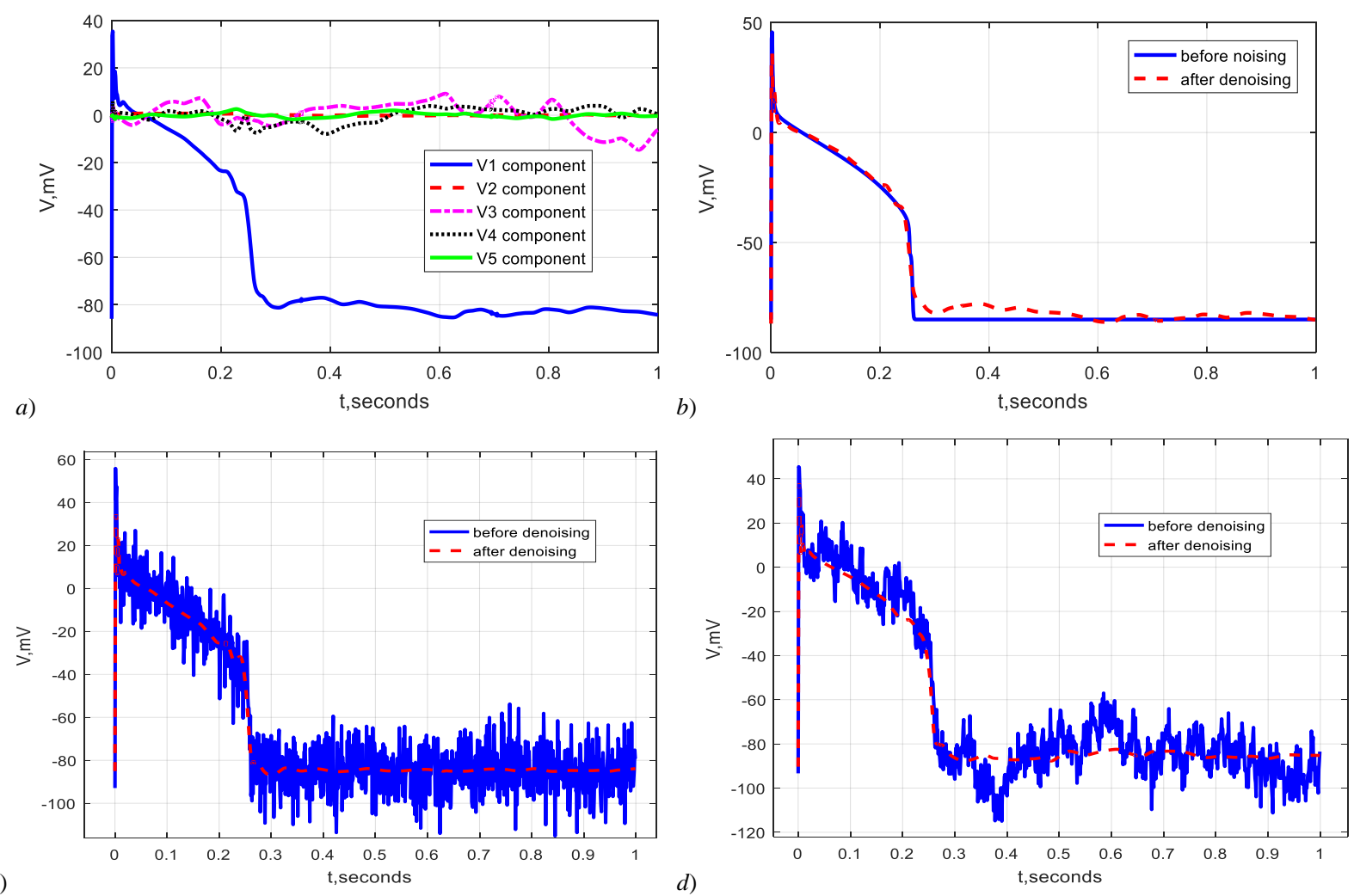

Fig. 6. Accumulation of 20 simulated cardiac action potentials, corrupted by pink (flicker) noise, with synchronization by the excitation pulses $\left(F_{s t}=1 \mathrm{~Hz}\right)$

were reconstructed with reduced background noise.

Despite the fairly good quality of noise elimination obtained by wavelets, some limitations were noticed. Denoising of cardiac cycles was performed with discarding only the portion of details by thresholding on each level so that steep fronts of AP pulses were not distorted. Comparison of original noised AP signals with $\mathrm{SNR}=30-60 \mathrm{~dB}$ and denoised AP signals demonstrated good noise suppression. However, for the noisy AP signals with $\mathrm{SNR}=10-30 \mathrm{~dB}$ noise suppression by wavelet transform was deficient.

The result of APs processing in low $\mathrm{SNR}=10 \mathrm{~dB}$ by means of the wavelet transform is presented in Fig. 5. Due to the similarity between the characteristics of background noise and target APs signal having very fast depolarization phase, de-noising of AP under low SNR condition is not always sufficient using only the wavelet transform.

Therefore, we propose a complex method, based both on wavelet transform and eigensubspaces analysis. For cardiac action potentials extraction from noised recordings and estimation of their morphology, wavelet transform should be applied as a tool for preliminary noise suppression and used in combination with eigensubspaces method.

Processing in the coordinate basis of eigenvectors distinguishes the main information about APs shape as signal subspace, reconstructed from the first principal eigenvector. The noise subspace with remaining noise components corresponds to the other eigenvectors (Fig. 6).

The assessment of noise removal quality was performed by comparing the values of relative root mean square error (RRMSE) in different signal to noise ratio for 2 techniques (Tables I and II):

$$
\text { RRMSE }=\sqrt{\frac{\sum_{i=0}^{n-1}\left(E_{i}-E_{i}{ }^{*}\right)^{2}}{\sum_{i=0}^{n-1} E_{i}^{2}}} \cdot 100 \%
$$

where $E_{i}$ is simulated APs sequence before white and pink noise superimposition, $E_{i}{ }^{*}$ is the denoised signal, $n$ is the length of the digital signal.

TABLE I. WAVELET DENOISING

\begin{tabular}{|c|c|c|c|}
\hline \multicolumn{2}{|c|}{ White noise } & \multicolumn{2}{c|}{ Pink noise } \\
\hline SNR, $\boldsymbol{d B}$ & $\boldsymbol{R R M S E , ~ \%}$ & SNR, $\boldsymbol{d B}$ & RRMSE, \% \\
\hline 10 & 3.51 & 10 & 11.72 \\
\hline 20 & 1.37 & 20 & 3.45 \\
\hline 30 & 0.51 & 30 & 1.09 \\
\hline 40 & 0.16 & 40 & 0.38 \\
\hline 50 & 0.05 & 50 & 0.11 \\
\hline 60 & 0.02 & 60 & 0.04 \\
\hline
\end{tabular}

Obtained results demonstrated good noise suppression (RRMSE $<1 \%$ ) in AP signals with $\mathrm{SNR}=40-60 \mathrm{~dB}$ for both techniques in the case of white and pink noise, and insufficient noise suppression (RRMSE $>1 \%$ ) in APs with $\mathrm{SNR}=10-30 \mathrm{~dB}$ for wavelet denoising. Moreover, in the case of pink noise sufficient noise suppression was achieved only by using the proposed complex method and the values of RRMSE are significantly different for both techniques with decreasing SNR. 
TABLE II. COMPLEX METHOD

\begin{tabular}{|c|c|c|c|}
\hline \multicolumn{2}{|c|}{ White noise } & \multicolumn{2}{c|}{ Pink noise } \\
\hline SNR, $\boldsymbol{d B}$ & RRMSE, \% & SNR, $\boldsymbol{d B}$ & RRMSE, \% \\
\hline 10 & 1.91 & 10 & 6.26 \\
\hline 20 & 0.91 & 20 & 2.38 \\
\hline 30 & 0.33 & 30 & 0.68 \\
\hline 40 & 0.11 & 40 & 0.24 \\
\hline 50 & 0.04 & 50 & 0.07 \\
\hline 60 & 0.02 & 60 & 0.03 \\
\hline
\end{tabular}

\section{CONCLUSIONS}

A novel approach for AP signal processing is proposed allowing for the detection of cardiac cycles under low SNR condition. The complex method is based on wavelet transform and processing in eigenvectors basis of cardiac cycles.

Simulation of the cardiac APs with noise was performed using parallel conductance model, which was improved by the introduction of an additional current source to the parallel branch. The results of computational experiments demonstrated the detection of cardiac APs without distortion for different SNR values.

The proposed method is aimed at detecting changes in AP signals and eliminating the noise artefacts, consequently, it is well suited to process signals obtained from experiments with hiPSC-CMs in lab-on-chip platforms for studying cardiomyocytes differentiation and drug screening.

\section{ACKNOWLEDGMENT}

The study was supported by EU-financed Horizon 2020 project AMMODIT (Approximation Methods for Molecular Modeling and Diagnosis Tools) - Grant Number MSCARISE 645672.

\section{REFERENCES}

[1] R. Plonsey and R. Barr, Bioelectricity. A Quantitative Approach, third ed. New York: Springer Science+Business Media, LLC, 2007. doi: 10.1007/978-0-387-48865-3_9.

[2] T. O'Hara, L. Virag, A, Varro, Y.Rudy, "Simulation of the undiseased human cardiac ventricular action potential: model formulation and experimental validation," PLoS Comput Biol., no.7, 2011, pp. 1-29.. doi: 10.1371/journal.pcbi.1002061.

[3] P. Liang, F. Lan, A.S.Lee, T. Gong, V. Sanchez-Freire, Y. Wang, S. Diecke, K. Sallam, J.W. Knowles, P.J. Wang, "Drug screening using a library of human induced pluripotent stem cell-derived cardiomyocytes reveals disease-specific patterns of cardiotoxicity," Circulation. 2013; 127:1677-1691. doi: 10.1161/CIRCULATIONAHA.113.001883
[4] F. Qian, C.Huang, Y.D. Lin, A.N. Ivanovskaya, T.J. O'Hara, R.H. Booth, C.J. Creek, H.A. Enright, D.A. Soscia, A.M. Belle, R. Liao, F.C. Lightstone, K.S. Kulp, E.K. Wheeler, "Simultaneous electrical recording of cardiac electrophysiology and contraction on chip." Lab Chip, 2017; doi: 10.1039/C7LC00210F.

[5] Junyi Ma, Liang Guo, Steve J.Fiene, Blake D. Anson, James A. Thomson, Timothy J. Kamp, Kyle L. Kolaja, Bradley J. Swanson and T. Craig, "High purity human-induced pluripotent stem cell-derived cardiomyocytes: electrophysiological properties of action potentials and ionic currents," Am J Physiol Heart Circ Physiol, 2011 Nov, 301(5), H2006-H2017. doi: 10.1152/ajpheart.00694.2011.

[6] E.S. Richardson, Y.F. Xiao, "Electrophysiology of Single Cardiomyocytes: Patch Clamp and Other Recording Methods," In: Sigg D., Iaizzo P., Xiao YF., He B. (eds) Cardiac Electrophysiology Methods and Models. 2010, Springer, Boston, MA. doi: 10.1007/978-1-4419-6658-2_16.

[7] Peter Lee, Matt Klos, Christian Bollensdorff, Lugia Hou, Paul Ewart, Timothy J. Kamp, Jianhua Zhang, Alexandra Bizy, Guadalupe Guerrero-Serna, Peter Kohl, Jose Jalife and Todd J. Herron, "Simultaneous Voltage and Calcium Mapping of Genetically Purified Human iPS Cell-Derived Cardiac Myocyte Monolayers," Circ Res, $2012 \quad$ June $8, \quad 110 \quad$ (12), pp.1556-1563. doi: $10.1161 /$ CIRCRESAHA.111.262535.

[8] M.J. Shattock, K.C. Park, H.Y. Yang, Lee A.W.C., S. Niederer, K.T. MacLeod, J. Winter, "Restitution slope is principally determined by steady-state action potential duration," Cardiovascular Research, Volume 113, Issue 7, 1 June 2017, 817-828. doi: 10.1093/cvr/cvx063.

[9] A.Pavesi, G.Adriani, M.Rasponi, I. Zervantonaki, G.B. Fiore, R.D. Kamm, "Controlled electromechanical cell stimulation on-a-chip.," Scientific Reports , 2015, 5, (11800),1-12. doi: 10.1038/srep11800.

[10] A. Marsano, C. Conficconi, M. Lemme, P. Occhetta, E. Gaudiello, E. Votta, G. Cerino, A. Redaelli, M. Rasponi, "Beating heart on a chip: a novel microfluidic platform to generate functional 3D cardiac microtissues," Lab Chip, no. 16, 2016, pp. 599-610. doi: 10.1039/c5lc01356a.

[11] N. Ivanushkina, K. Ivanko, Y. Prokopenko, V.Tymofieiev, R.Visone $\mathrm{R}$, and A. Redaelli, "Computer modelling of cardiac cells electrical activity," In Proceedings of 2018 IEEE 38th International Scientific Conference on Electronics and Nanotechnology (ELNANO), '04 2018, pp. 369-374. doi: 10.1109/ELNANO.2018.8477567

[12] Robert M. Miura, "Analysis of excitable cell models," Journal of Computational and Applied Mathematics. Vol 144, Issues 1-2, 2002, pp. 29-47. doi: 10.1016/S0377-0427(01)00550-7.

[13] Charles F. Stevens, "Inferences about Membrane Properties from Electrical Noise Measurements," Byophis J., 1972, 12(8), 1028-1047, doi: 10.1016/S006-3495(72)86141-1.

[14] N.G. vanushkina, K.O.Ivanko, V.I. Timofeyev, "Analysis of lowamplitude signals of cardiac electrical activity," Radioelectronics and Communications Systems (distributed by Springer), vol.57, no.10, 2014, p.465-473. doi: 10.3103/S0735272714100057.

[15] K.H. Kim, S.J. Kim, "A wavelet-based method for action potential detection from extracellular neural signal recording with low signalto-noise ratio," IEEE Trans Biomed Eng, 2003, Aug, 50 (8), pp. 9991011.

[16] H.Nakatani, T.Watanabe, N.Hoshimiya, "Detection of nerve AP under low signal-to-noise ratio condition," IEEE Trans Biomed Eng, 2001, Aug, 48 (8), pp. 845-849. doi: 10.1109/10.936360.

[17] The phisiological mesurement handbook/Edited by John G. Webster. CRC Press. 2015, 590 p. ISBN-13:978-1-4398-0848-1. 